\title{
Natural concurrent infection of Vibrio harveyi and V. alginolyticus in cultured hybrid groupers in Malaysia
}

\begin{abstract}
In September 2016, a marine fish farm operator in Selangor, Malaysia, reported a disease outbreak affecting juvenile hybrid groupers (Camouflage Grouper Epinephelus polyphekadion $\times$ Tiger Grouper E. fuscoguttatus). The average daily mortality was 120 fish, resulting in a cumulative mortality rate of $29 \%$ within $10 \mathrm{~d}$. The affected hybrid groupers displayed lethargy, excessive mucus production, rotten fins, congestion of livers and kidneys, and enlargement of spleens. Microscopically, general congestion of the brains and internal organs was evident. Vibrio harveyi and V. alginolyticus were successfully isolated from the diseased fish. The isolated pathogens were found to be sensitive to oxytetracycline and tetracycline, but resistant towards ampicillin and vancomycin. Experimental infections using the isolated V. harveyi (108 CFU/mL), V. alginolyticus $(108 \mathrm{CFU} / \mathrm{mL})$, and concurrent infection by V. harveyi (108 CFU/mL) and V. alginolyticus (108 CFU/mL) in juvenile Asian Seabass Lates calcarifer resulted in 60, 100, and 100\% mortality, respectively, within $240 \mathrm{~h}$ postinfection. The experimentally infected Asian Seabass demonstrated similar clinical signs and histopathological changes as the naturally infected hybrid groupers. However, concurrently infected fish demonstrated severe clinical signs and histopathological changes compared with single infections. These results suggest that both isolates of Vibrio are pathogenic to fish and responsible for the disease outbreak. However, concurrent infection involving $\mathrm{V}$. alginolyticus and $\mathrm{V}$. harveyi leads to a more devastating impact to the cultured fish. This is the first report of concurrent Vibrio infection in cultured marine fish in Malaysia.
\end{abstract}

